\title{
Prevotella and Porphyromonas infections in children
}

I. BROOK

Department of Pediatrics, Georgetown University School of Medicine, Washington, DC, USA

\begin{abstract}
Summary. From 1974 to 1994, 504 isolates of Prevotella and Porphyromonas spp. were obtained from $435(21 \%)$ of 2033 specimens from 418 children. They included $160(32 \%) \mathrm{Pr}$. melaninogenica, $105(21 \%)$ Pr. intermedia, $84(17 \%)$ P. asaccharolytica, $58(12 \%)$ Pr. orisbuccae, and $58(12 \%)$ Pr. oralis. Most Prevotella and Porphyromonas spp. were isolated from abscesses (176), pulmonary infections (85), ear infections (82), wound infections (44), peritonitis (38), paronychia (15) and chronic sinusitis (14). Predisposing conditions were noted in $111(27 \%)$ of the cases; these included previous surgery in $41(10 \%)$, foreign body in $36(9 \%)$, neurological deficiencies in $29(7 \%)$, immunodeficiency in $21(5 \%)$, steroid therapy in $12(4 \%)$, diabetes in $8(2 \%)$ and malignancy in $7(2 \%)$. Prevotella and Porphyromonas spp. were the only isolates in $14(3 \%)$ patients, and mixed infection was encountered in 404 (97\%). The micro-organisms most commonly isolated with Prevotella and Porphyromonas spp. were anaerobic cocci (393 isolates), Fusobacterium spp. (108), Bacteroides spp. (B. fragilis group) (95), Escherichia coli (56) and other gram-negative anaerobic bacilli (52). Most Bacteroides spp. and E. coli were isolated from intra-abdominal infections and skin and soft tissue infections around the rectal area, whereas most Fusobacterium spp. were isolated from oropharyngeal, pulmonary and head and neck sites. $\beta$ Lactamase production was detected in 191 (38\%) Prevotella and Porphyromonas isolates from all body sites. All patients received antimicrobial therapy, and surgical drainage was performed in $173(41 \%)$ cases. Four patients died from their infection. These data illustrate the spectrum and importance of Prevotella and Porphyromonas spp. in infections in children.
\end{abstract}

\section{Introduction}

Growing interest has been generated in recent years about the role of anaerobic bacteria in paediatric infections. ${ }^{1}$ A group of organisms that are implicated in anaerobic infections arising from the respiratory, genital and gastrointestinal tracts are the gram-negative anaerobic bacilli of the genera Prevotella and Porphyromonas. ${ }^{2}$ However, the incidence and significance of these infections remain poorly defined because of variations in diagnostic methods and nonadherence to proper anaerobic culture techniques among microbiology laboratories. Furthermore, DNA homology studies have caused nomenclature changes in the names of these organisms. ${ }^{3}$ Pr. intermedia, Pr. melaninogenica and P. asaccharolytica, all known as "pigmented bacteroides", were previously classified as subspecies of Bacteroides melaninogenicus, and Pr. oralis and Pr. oris-buccae were previously called Bacteroides spp.
The role of Prevotella and Porphyromonas spp. in infections in children has become more important in recent years because of the steady increase in penicillin resistance in these species through the production of $\beta$ lactamase; this was first noticed in the late 1970s. ${ }^{4}$

This retrospective review summarises experience of the past 20 years in the isolation of Prevotella and Porphyromonas spp. from infections in children. Some of the data have been published before in articles describing the role of anaerobic bacteria in various paediatric infections, ${ }^{5-20}$ but cases not previously presented are also included and the unique nature of infections with Prevotella and Porphyromonas spp. in children is emphasised.

\section{Patients and methods}

\section{Patients}

The specimens included in this review were studied or reviewed by the author between June 1974 and June 1994. They were collected in the following hospitals: 
University of California Medical Center, Los Angeles County Medical Center, and Serra Memorial Hospital in Los Angeles, and Fairview State Hospital, Costa Mesa, CA; Children's Hospital National Medical Center and South-East Community Medical Center, Washington, DC; and the Naval Hospital in Bethesda, MD.

The clinical microbiology laboratories' records were reviewed to identify patients with infections from whom Prevotella and Porphyromonas spp. were isolated. The available case histories of all patients from whom these organisms had been isolated were reviewed to ascertain the presence and site of infection, associated micro-organisms, underlying disease process and possible predisposing or associated conditions.

\section{Microbiological examination}

Only specimens that were properly collected without contamination by the normal skin or mucosal flora and submitted in transport media appropriate for anaerobic bacteriological investigations were accepted by the microbiology laboratories. These were generally specimens obtained during surgery or by aseptic needle or biopsy aspiration of abscesses or fluid from body cavities. Lung aspirates were obtained by transtracheal aspiration, or through a tracheostomy or endotracheal tube, or by biopsy. Urine was collected by suprapubic aspiration. When possible, pus and fluids were collected and transported in syringes. Tissues were transported in oxygen-free gassed-out tubes. Swab specimens were submitted in the Port-a-Cul transport swab system (BBL, Cockeysville, MD, USA). However, precise records of all of the transport media used was not available. Blood was collected aseptically from patients suspected of having bacteraemia and was inoculated $(10 \%)$ into one bottle each of two commercially produced blood culture broth media, both under vacuum and with $\mathrm{CO}_{2} 5 \%$ in the atmosphere.

The specimens were inoculated on to pre-reduced vitamin $\mathrm{K}_{1}$-enriched Brucella Blood Agar (BBL), blood agar with kanamycin $75 \mathrm{mg} / \mathrm{L}$ and vancomycin $7.5 \mathrm{mg} / \mathrm{L}$, a blood agar plate containing colistin sulphate $0.01 \mathrm{mg} / \mathrm{L}$ and nalidixic acid $50 \mathrm{mg} / \mathrm{L}$, and an enriched thioglycolate broth containing haemin $5 \mathrm{mg} / \mathrm{L}$ and vitamin $\mathrm{K}_{1} 0 \cdot 1 \mathrm{mg} / \mathrm{L}^{21,22}$ The cultures were incubated in GasPak jars (BBL) and examined after 48 and $96 \mathrm{~h}$.

Plates that showed any growth were held until the micro-organisms had been identified. All cultures that showed no growth were held for at least 5 days. Microorganisms were identified by the API anaerobic system (Analytab Products, Plainview, NY, USA) or by the Minitek system (BBL). In addition to these tests, when complete identification was not possible by the above methods, other carbohydrate utilisation or fermentation tests (Scott Laboratories, Fiskeville, RI, USA) and gas-liquid chromatography ${ }^{22}$ were performed as needed to identify the organisms. The criteria for identification were according to guidelines previously published..$^{21,22} \beta$-Lactamase activity was determined on all isolates by the chromogenic cephalosporin analogue $87 / 312$ method. ${ }^{23}$

\section{Results}

\section{Patients}

From a total of 2033 specimens accepted as suitable for examination for anaerobic bacteria, 504 isolates of Prevotella and Porphyromonas spp. were obtained from $435(21.4 \%)$ specimens from 418 patients. Patients' ages ranged from 10 days to 18 years (mean 7 years 8 months); 246 were male and 172 female. Clinical records were not available from another 26 cases; these were not included in the final analysis.

Predisposing or underlying conditions were noted in $111(27 \%)$ cases. A single underlying condition was noted in 64 cases, two such conditions were recorded in 42 and three in five; these were previous surgery ( 41 ; $10 \%$ of all patients), presence of a foreign body (36; $9 \%$ ), neurological deficiency $(29 ; 7 \%)$, immunodeficiency $(21 ; 5 \%)$, steroid therapy $(12 ; 3 \%)$, diabetes $(8 ; 2 \%)$, malignancy $(7 ; 2 \%)$, sickle cell disease $(5$; $1 \%)$ and trauma $(4 ; 1 \%)$.

\section{Prevotella and Porphyromonas isolates}

The Prevotella and Porphyromonas isolates comprised 160 (32\%) Pr. melaninogenica, 105 (21\%) Pr. intermedia, $84(17 \%)$ P. asaccharolytica, $58(11 \%)$ Pr. oris-buccae, $58(11 \%)$ Pr. oralis and $29(8 \%)$ Prevotella spp. (tables I and II).

Infections were polymicrobial in 404 patients (97\%), but in $14(3 \%)$ a Prevotella or a Porphyromonas sp. was isolated in pure culture (table III). Most Prevotella and Porphyromonas isolates were from abscesses $(176$ of $504 ; 35 \%)$, pulmonary infections $(85 ; 17 \%)$, ear infections $(82 ; 16 \%)$, wounds $(44$; $9 \%)$, peritonitis $(38 ; 8 \%)$ and chronic sinusitis $(14$; $3 \%)$.

There were 1229 isolates of other genera with the Prevotella and Porphyromonas spp. in the 421 specimens from mixed infections (tables I and II); 841 $(68 \%)$ of these were strict anaerobes and $388(32 \%)$ were facultative or aerobic species. The number of isolates in the mixed culture was in the range $2-8$ (average 3.0 isolates/specimen; $2 \cdot 1$ anaerobes and 0.9 facultative or aerobic species). The anaerobic microorganisms isolated most commonly with Prevotella and Porphyromonas spp. were: Peptostreptococcus spp. (393 isolates), Fusobacterium spp. (108, including $62 F$. nucleatum, 23 F. necrophorum and 11 F. mortiferum), Bacteroides spp. (previously called the B. fragilis group) (95, including 46 B. fragilis, 24 $B$. distasonis, 10 each of $B$. thetaiotaomicron and $B$. vulgatus and five $B$. uniformis), other gram-negative 


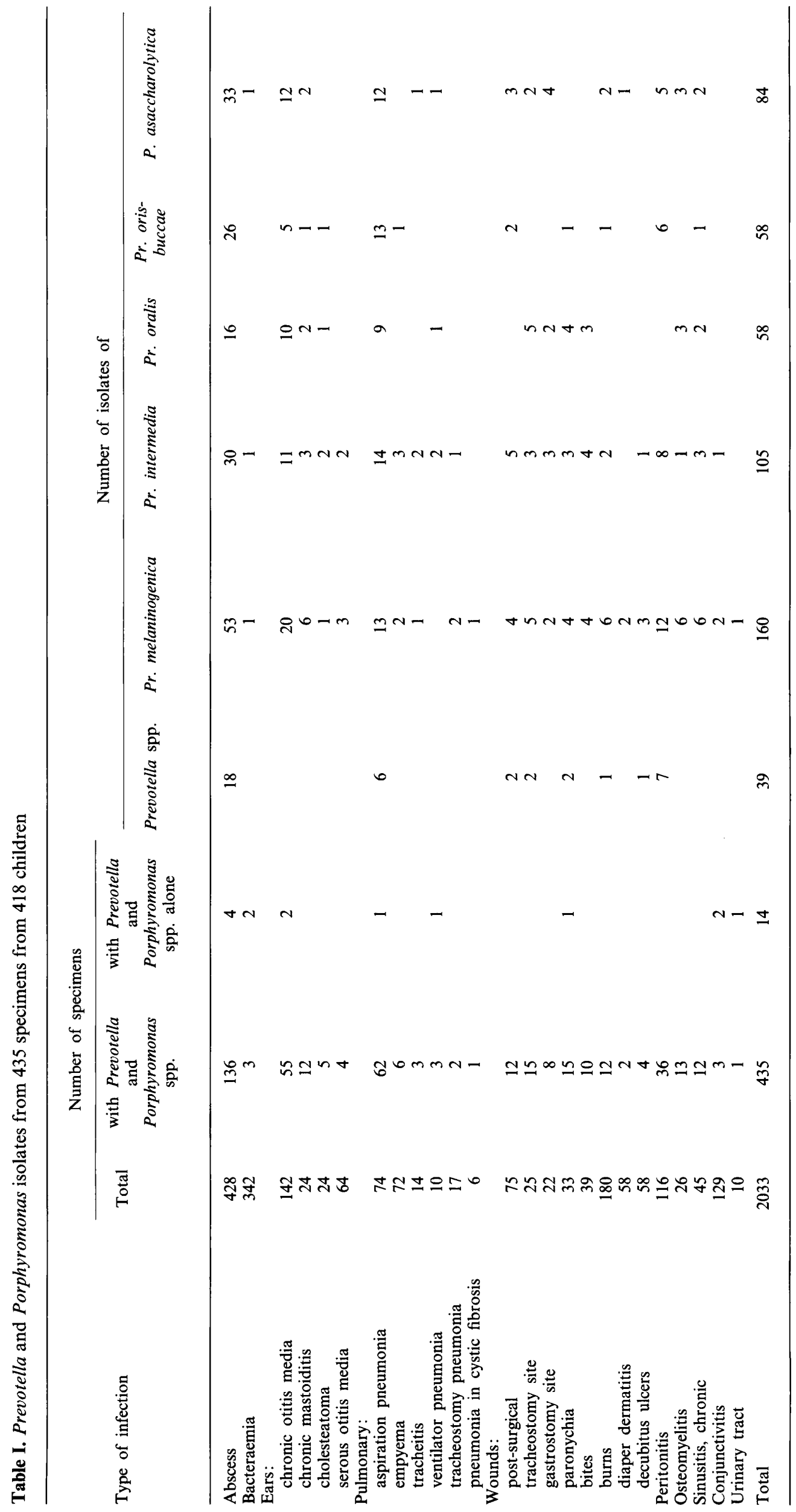




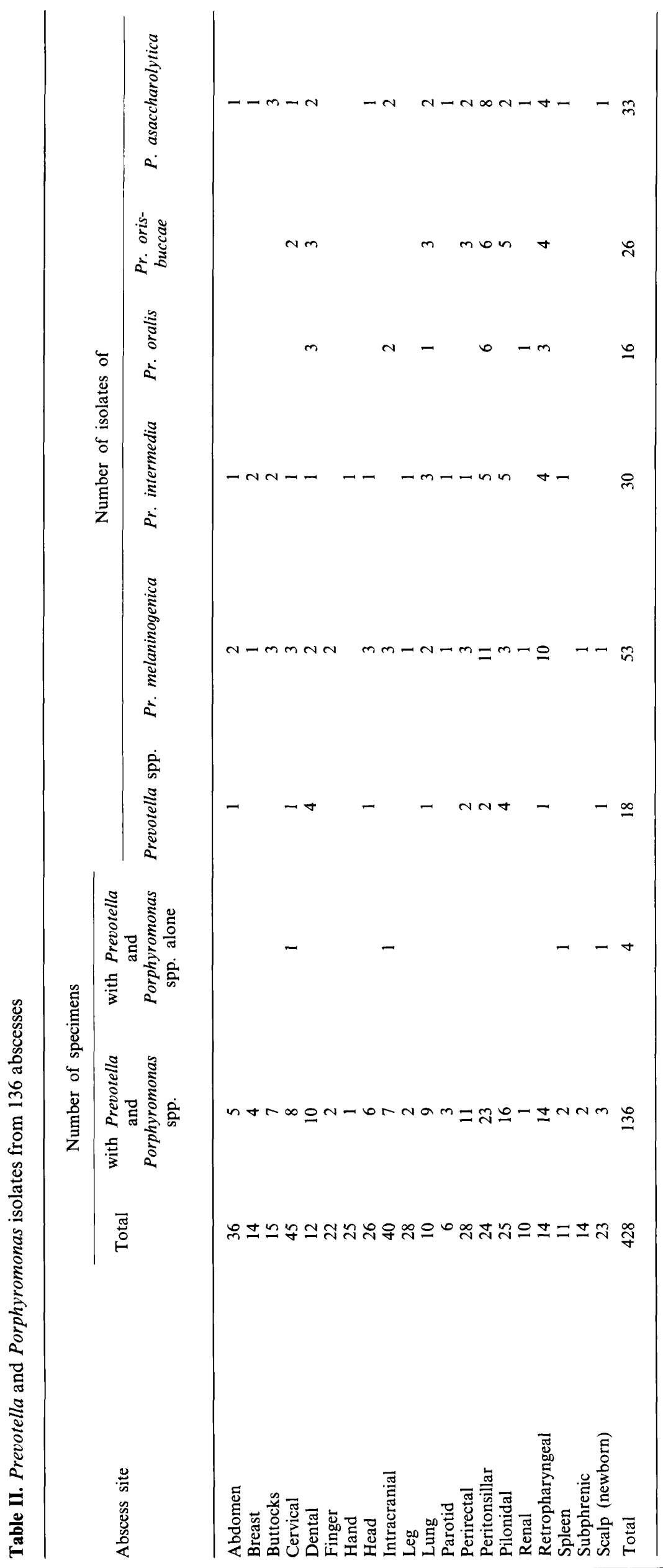




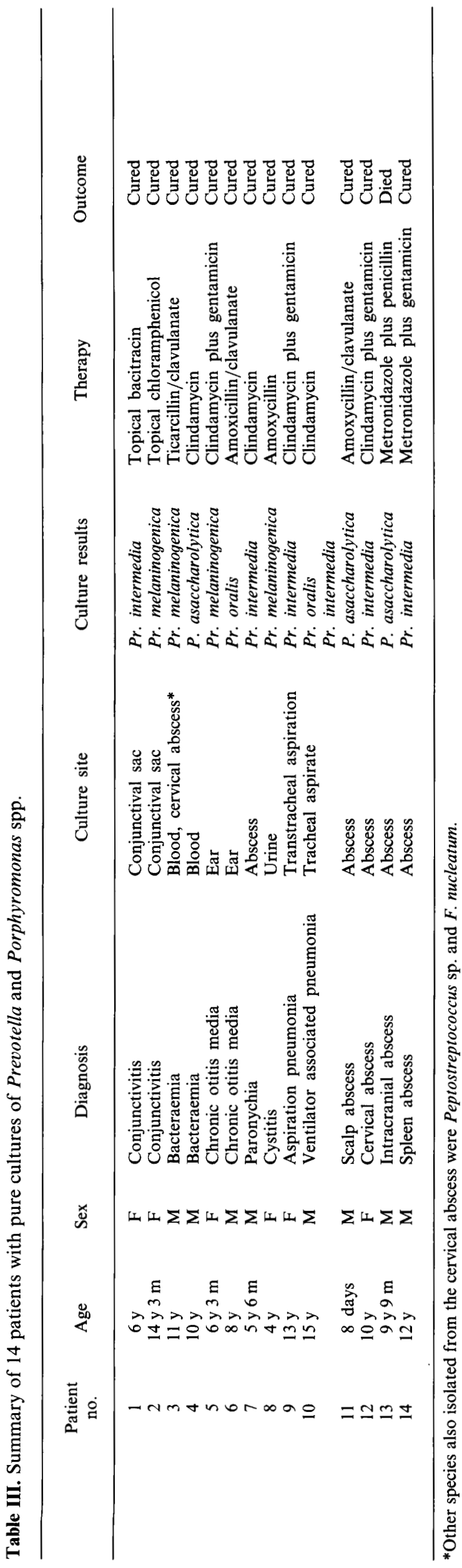

anaerobic bacilli (52), Clostridium spp. (42), Eubacterium spp. (26), Veillonella spp. (19), Bifidobacterium spp. (7) and Actinomyces spp. (3). The most common aerobic and facultative organisms isolated with Prevotella and Porphyromonas spp. were: $\alpha$ haemolytic streptococci (61), Escherichia coli (56), non-haemolytic streptococci (50), Staphylococcus aureus (49), Klebsiella pneumoniae (36), Haemophilus influenzae (33), H. parainfluenzae (28), Pseudomonas aeruginosa (27), Streptococcus pyogenes (15) and Str. pneumoniae (14). Most Bacteroides and E. coli isolates mixed with Prevotella and Porphyromonas spp. were from intra-abdominal infections and skin and soft tissue infections around the rectal area, whereas most Fusobacterium spp. and Haemophilus spp. were from oropharyngeal, pulmonary, and head and neck infections. Anaerobic cocci were isolated from all sites.

$\beta$-Lactamase production. $\beta$-Lactamase production was detected in $191(38 \%)$ Prevotella and Porphyromonas isolates; the proportion was similar from all body sites. The incidence of $\beta$-lactamase production was similar among isolates of all Prevotella and Porphyromonas spp.

\section{Types of infection}

Abscesses. Of the 132 patients with abscesses with mixed infections (table II), the most common abscesses from which Prevotella and Porphyromonas spp. were isolated were peritonsillar (23), pilonidal (16), retropharyngeal (14), perirectal (11), dental (10), lung (9), cervical (7), intracranial (6) and buttocks (7). The most common other anaerobic isolates from these abscesses with Prevotella and Porphyromonas spp. were Peptostreptococcus spp. (128 isolates), Fusobacterium spp. (63), Bacteroides spp. (29), Clostridium spp. (18) and Veillonella spp. (14). The most common aerobic and facultative organisms isolated with Prevotella and Porphyromonas spp. were $\alpha$-haemolytic streptococci (38), E. coli (26), non-haemolytic streptococci (25), $S$. aureus (24), and Haemophilus spp. (6). The distribution of these organisms in abscesses at various sites was as described in the previous paragraph.

A single $P$. asaccharolytica isolate was from a blood culture; it was mixed with Str. pyogenes and was from a patient with peritonsillar abscess. Similar organisms were also isolated from the abscess. However, the abscess also contained $F$. nucleatum and Pstr. magnus.

Ear infections. Eighty isolates of Prevotella and Porphyromonas were from chronic and serous otitis media, cholesteatoma and chronic mastoiditis and were mixed with Fusobacterium spp., Peptostreptococcus spp., S. aureus and Ps. aeruginosa.

Pulmonary infections. Eighty-two Prevotella and Porphyromonas isolates were from pulmonary sites. They were mostly mixed with Fusobacterium spp., Peptostreptococcus spp. and enterobacteria. An associated empyema was present in seven of the patients with aspiration pneumonia, and similar Prevotella and Porphyromonas spp. were also isolated 
from that site. Six isolates were from empyema, in four cases associated with aspiration pneumonia (five isolates) and in one case associated with a head and neck infection.

Wound infections. The 16 Prevotella and Porphyromonas isolates from post-surgical wounds were from nine wounds of the head and neck and three followed abdominal procedures. In all cases, they were mixed with other bacteria-Fusobacterium spp., Peptostreptococcus spp., S. aureus, Str. pyogenes and Str. pneumoniae were mostly found in head and neck wounds, and E. coli, Clostridium spp. and $B$. fragilis in abdominal wounds. The 11 Prevotella and Porphyromonas isolates from tracheostomy site wounds were mixed with Peptostreptococcus spp., Fusobacterium spp. and S. aureus. The 18 isolates from gastrostomy site wound were mixed mostly with enterobacteria, $S$. aureus and Bacteroides spp. Fourteen paronychia isolates were mostly mixed with S. aureus and Fusobacterium spp. In one instance the Prevotella sp. was also isolated from an associated digital osteomyelitis.

Eleven Prevotella and Porphyromonas isolates were from bites, three from human bites and eight from animal bites. They were isolated from bites at all body sites and were mostly mixed with Peptostreptococcus and Streptococcus spp. Twelve isolates were from burns, five from burns on the trunk, four from facial burns and three from burns around the rectal area.

The three isolates from diaper dermatitis were mixed with B. fragilis, Str. pyogenes and E. coli and the four Prevotella and Porphyromonas isolates from decubitus ulcers were from the head and neck region.

Peritonitis. The 38 peritoneal isolates of Prevotella and Porphyromonas spp. were from 36 children, 25 with a ruptured viscus, six with spontaneous peritonitis and four following abdominal trauma. They were mostly isolated mixed with Peptostreptococcus spp., Bacteroides spp. and enterobacteria.

Osteomyelitis. The 13 isolates of Prevotella and Porphyromonas spp. were mostly mixed with Peptostreptococcus and Fusobacterium spp. They were isolated from two cases each of osteomyelitis of the temporal bone (associated with mastoiditis), frontal bone (associated with frontal sinusitis), maxilla (with maxillary sinusitis), mandible (associated with a periodontal abscess), and one each of the occipital and temporal bone (associated with decubitus and diabetic ulcers), and metacarpal bone (associated with paronychia).

Sinus infections. The 17 Prevotella and Porphyromonas isolates from sinuses were from the maxillary (13 isolates), frontal (3) and sphenoid (1) sinuses. In all cases, they were mixed, mostly with Fusobacterium and Peptostreptococcus spp.

Conjunctivitis. A single Pr. melaninogenica isolate was from the conjunctiva, mixed with an $\alpha$-haemolytic streptococcus and was from a patient who wore contact lenses.
Clinical and microbiological data of the 14 patients from whose cultures only Prevotella and Porphyromonas spp. were grown are listed in table III. Four patients had abscesses, two each conjunctivitis, bacteraemia, chronic otitis media and pneumonia, and one each cystitis and paronychia. One patient (no. 3) with bacteraemia had cervical adenitis from which similar organisms were isolated. Of the two cases with conjunctivitis, one wore contact lenses, and the other had no known predisposing condition. The patients with chronic otitis media (no. 5) and aspiration pneumonia (no. 9) had Down's syndrome. One patient (no. 13) with intracranial abscess that developed after frontal sinusitis died of the infection.

\section{Treatment and outcome}

Antimicrobial therapy was administered to all patients with surgical drainage or correction of pathology in $173(41 \%)$ instances. The antimicrobial agents administered were a penicillin in 132 cases, aminoglycosides in 126 , clindamycin in 83 , a cephalosporin in 71, erythromycin in 33, chloramphenicol in 21 , metronidazole in 19 and co-trimoxazole in five. Four $(1 \%)$ patients died of their infection. The deaths occurred in one case each of lung abscess (mixed with $K$. pneumoniae), perirectal abscess (mixed with $E$. coli and $B$. fragilis), peritonitis (mixed with $E$. coli) and intracranial abscess (table III; no. 13).

\section{Discussion}

This retrospective review demonstrates the prevalence of Prevotella and Porphyromonas spp. in various infections in children. The 504 Prevotella and Porphyromonas isolates were from $435(21 \%)$ of 2033 specimens submitted for culture for anaerobic bacteria. Prevotella and Porphyromonas spp. are especially prevalent in abscesses (mostly of the head and neck and intracranial), chronic otitis media, aspiration pneumonia, peritonitis and sinusitis. Although most Prevotella and Porphyromonas isolates were mixed with other aerobic and anaerobic bacteria, their cultivation as the only isolate in $14(3 \%)$ of the patients (table III) illustrates their potential virulence and clinical significance.

Prevotella and Porphyromonas spp. are part of the normal oral, gastrointestinal and female genital tract flora. ${ }^{24,25}$ Therefore, these sites are the most common origin of infections that involve these organisms. Prevotella and Porphyromonas spp. are often isolated from mixed infections with other anaerobes and aerobic or facultative species that also originate from these sites. This is the most plausible reason why enterobacteria and Bacteroides spp. are the most common isolates mixed with Prevotella and Porphyromonas spp. in abdominal infections or infections associated with gastrointestinal flora. Simi- 
larly, Fusobacterium spp., Str. pneumoniae and Haemophilus spp. predominate in mixed infections that originate from the oral flora.

Support for the pathogenic role of Prevotella and Porphyromonas spp. can be derived from in-vivo studies that demonstrated their synergic potential with other anaerobic and aerobic bacteria. ${ }^{26,27}$ With few exceptions, Prevotella and Porphyromonas spp. enhanced the growth of other aerobic and anaerobic bacteria. Bacterial synergy was demonstrated between Prevotella and Porphyromonas spp. and Peptostreptococcus spp., Fusobacterium spp., S. aureus and Ps. aeruginosa in a subcutaneous abscess model in mice. ${ }^{27}$ In this model, Prevotella and Porphyromonas spp. enhanced the development of abscesses and increased mortality when they were co-inoculated with the other organisms. As was found with other gramnegative anaerobic bacilli, the ability of Prevotella and Porphyromonas spp. to induce abscesses was correlated with the presence of a mucopolysaccharide capsule. ${ }^{26}$ The presence of capsular material suppresses phagocytosis and, therefore, is an important factor influencing the pathogenicity of Prevotella and Porphyromonas spp. ${ }^{28}$ The production of subcutaneous abscesses in experimental animals could be an indication of the organism's virulence, as has been noted in other anaerobic species. ${ }^{26}$

Prevotella and Porphyromonas spp. can be isolated from various infections of man. They can be isolated from $20-30 \%$ of all anaerobic infections in children ${ }^{29-31}$ and $10 \%$ of anaerobic bacteraemia., ${ }^{1,2}$ The common infections they cause in children are upper respiratory tract and head and neck infections. However, in older individuals they tend to induce lower respiratory infection as a result of aspiration.

The spectrum of infections with Prevotella and Porphyromonas spp. in adults and children includes bactaeremia (mostly of oropharyngeal origin), head and neck infections (such as chronic otitis media,

\section{References}

1. Brook I. Pediatric anaerobic infections: diagnosis and management, 2nd edn. St. Louis, CV Mosby Co. 1989.

2. Finegold SM. Anaerobic bacteria in human disease. New York, Academic Press. 1977

3. Van Steenbergen TJM, Van Winkelhoff AJ, de Graaf J. Classification and typing methods of black-pigmented gram-negative anaerobes. FEMS Immunol Med Microbiol $1993 ; 6: 83-88$.

4. Brook I, Calhoun L, Yocum P. Beta-lactamase-producing isolates of Bacteroides species from children. Antimicrob Agents Chemother 1980; 18: 164-166.

5. Brook I. Chronic otitis media in children: microbiological studies. Am J Dis Child 1980; 134: 564-566.

6. Brook I. Bacteriologic features of chronic sinusitis in children. JAMA 1981 ; 246 : 967-969.

7. Brook I, Controni G, Rodriguez WJ, Martin WJ. Anaerobic bacteremia in children. Am $J$ Dis Child 1980; 134: $1052-1056$

8. Brook I, Grimm S, Kielish RB. Bacteriology of acute periapical abscess in children. $J$ Endodont 1981 ; $7: 378-380$.

9. Brook I. Aerobic and anaerobic bacteriology of peritonsillar abscess in children. Acta Pediatr Scand 1981; 70: 831-835.

10. Brook I. Aerobic and anaerobic bacteriology of chronic mastoiditis in children. Am J Dis Child $1981 ; 135$ : 478-479. sinusitis and mastoiditis, peritonsillar and retropharyngeal abscesses, gingival and dental infections), pulmonary infections (aspiration pneumonia, lung abscess and empyema), intracranial infections (meningitis and intracranial abscess), gastrointestinal infections (peritonitis and hepatic and abdominal abscess), osteomyelitis, uro-genital (prostatic and female genital abscess and amnionitis), and skin and soft tissue infections especially around the oropharyngeal area. ${ }^{1,2}$

The management of infection with Prevotella and Porphyromonas spp. includes medical and surgical approaches. Surgical drainage of pus and debridement of necrotic tissue are an integral part of the therapeutic approach, and should accompany administration of proper antimicrobial therapy.

Prevotella and Porphyromonas spp. were generally susceptible to various antimicrobial agents that include penicillins and cephalosporins. ${ }^{32}$ However, within the past two decades an increase in the rate of $\beta$ lactamase-producing Prevotella and Porphyromonas spp. has been observed. ${ }^{4}$ The rate of recovery of these $\beta$-lactamase-producing strains was $38 \%$ among the isolates reported in this study. $\beta$-Lactamase-producing strains may inactivate penicillin, protecting themselves as well as other bacteria mixed with them in polymicrobial infections ${ }^{33}$ The addition of a $\beta$-lactamase inhibitor (e.g., clavulanic acid) to penicillins makes these agents effective against these resistant organisms. ${ }^{34}$ Other antimicrobial agents effective against $\beta$-lactamase-producing Prevotella and Porphyromonas spp. include clindamycin, metronidazole, cefoxitin, chloramphenicol and imipenem. ${ }^{1,2}$

The laboratory assistance of the staff of the microbiology laboratories at the University of California Medical Center, Los Angeles County Medical Center, and Serra Memorial Hospital in Los Angeles, and Children's Hospital National Medical Center and South-East Medical Center in Washington, D.C., and the Naval Hospital in Bethesda, MD. and the secretarial support of Sarah Blaisdell and Joanie Pietrafitta are gratefully acknowledged.

11. Brook I. Aerobic and anaerobic bacteriology of cervical adenitis in children. Clin Pediatr 1980; 19: 693-696.

12. Brook I. Aerobic and anaerobic bacteriology of intracranial abscesses. Pediatr Neurol 1992; 8: 210-214.

13. Brook I. Aerobic and anaerobic bacteriology of cholesteatoma. Laryngoscope $1981 ; 91$ : 250-253.

14. Brook I. Microbiology of retropharyngeal abscesses in children. Am J Dis Child 1987; 141: 202-204.

15. Brook I, Friedman E, Rodriguez WJ, Controni G. Complications of sinusitis in children. Pediatrics 1980; 66: 568-572.

16. Brook I. Aerobic and anaerobic bacterial flora of acute conjunctivitis in children. Arch Ophthalmol 1980; 98: 833-835.

17. Brook I. Bacterial studies of peritoneal cavity and postoperative surgical wound drainage following perforated appendix in children. Ann Surg 1980; 192: 208-212.

18. Brook I, Finegold SM. Bacteriology of aspiration pneumonia in children. Pediatrics 1980; 65: 1115-1120.

19. Brook I, Randolph JG. Aerobic and anaerobic bacterial flora of burns in children. $J$ Trauma $1981 ; 21$ : 313-318.

20. Brook I, Finegold SM. Bacteriology and therapy of lung abscess in children. $J$ Pediatr 1979; 94: 10-12.

21. Sutter VL, Citron DM, Edelstein MAC, Finegold SM. Wadsworth anaerobic bacteriology manual, 4th edn. Belmont, CA, Star Publishing Co., 1985 
22. Holdeman LV, Cato EP, Moore WEC. Anaerobe laboratory manual, 4th edn. Virginia Polytechnic Institute and State University, Blacksburg. 1977.

23. O'Callaghan CH, Morris A, Kirby SM, Shingler AH. Novel method for detection of beta-lactamases by using a chromogenic cephalosporin substrate. Antimicrob Agents Chemother 1972; 1: 283-288.

24. Socransky SS, Manganiello SD. The oral microbiota of man from birth to senility. J Periodontol 1971; 42: 485-496.

25. Rosebury T. Microorganisms indigenous to man. New York, McGraw-Hill Book Co. 1962.

26. Brook I, Gillmore JD, Coolbaugh JC, Walker RI. Pathogenicity of encapsulated Bacteroides melaninogenicus group, $B$. oralis and $B$. ruminicola subsp. brevis in abscesses in mice. $J$ Infect 1983; $7: 218-226$.

27. Brook I, Hunter V, Walker RI. Synergistic effect of Bacteroides, Clostridium, Fusobacterium, anaerobic cocci, and aerobic bacteria on mortality and induction of subcutaneous abscesses in mice. $J$ Infect Dis 1984; 149: 924-928.

28. Ingham HR, Tharagonnet D, Sisson PR, Selkon JB, Codd AA.
Inhibition of phagocytosis in vitro by obligate anaerobes. Lancet 1977 ; 2: 1252-1254.

29. Dunkle LM, Brotherton TJ, Feigin RD. Anaerobic infections in children: a prospective study. Pediatrics 1976; 57: 311-320.

30. Thirumoorthi MC, Keen BM, Dajani AS. Anaerobic infections in children: a prospective survey. J Clin Microbiol 1976; 3: 318-323.

31. Brook I, Martin WJ, Cherry JD, Sumaya CV. Recovery of anaerobic bacteria from pediatric patients. A one-year experience. Am J Dis Child 1979; 133: 1020-1024.

32. Sutter VL, Finegold SM. Susceptibility of anaerobic bacteria to 23 antimicrobial agents. Antimicrob Agents Chemother $1976 ; 10: 736-752$.

33. Brook I. The role of beta-lactamase-producing bacteria in the persistence of streptococcal tonsillar infection. Rev Infect Dis 1984; 6: 601-607.

34. Appelbaum PC, Spangler SK, Jacobs MR. Susceptibilities of 394 Bacteroides fragilis, non-B. fragilis group Bacteroides species, and Fusobacterium species to newer antimicrobial agents. Antimicrob Agents Chemother 1991;35: 1214-1218. 